\title{
Surgical intervention for renal cell carcinoma
}

\section{with inferior vena cava extension combined with laparoscopic procedure}

This article was published in the following Dove Press journal:

Research and Reports in Urology

19 June 2017

Number of times this article has been viewed

\author{
Toshio Kamimura' \\ Kazutaka Kida' \\ Masashi Takeda' \\ Shunsuke Sato' \\ Masato Fujii' \\ Masahiro Inoue' \\ Hiromasa Tsukino' \\ Shoichiro Mukai' \\ Atsushi Nanashima ${ }^{2}$ \\ Kunihide Nakamura ${ }^{2}$ \\ Toshiyuki Kamoto' \\ 'Department of Urology, Faculty of \\ Medicine, University of Miyazaki, \\ Miyazaki, Japan; ${ }^{2}$ Department \\ of Surgery, Faculty of Medicine, \\ University of Miyazaki, Miyazaki, Japan
}

Correspondence: Shoichiro Mukai Department of Urology, Faculty of Medicine, University of Miyazaki, 5200 Kihara, Kiyotake, Miyazaki 889-1692, Japan

Email syoichiro_mukai@med.miyazaki-u. ac.jp

\begin{abstract}
Clear cell renal cell carcinoma (ccRCC) shows extreme hypervascularity, which may cause significant bleeding during surgery. For this reason, control of arterial blood supply is an important factor in the choice of operative procedure and in avoiding perioperative complications. This case series reports the successful dissection of renal artery in the preliminary stage of laparoscopic procedure in three ccRCC patients with inferior vena cava (IVC) extension. Patient 1 had right renal cell carcinoma (RCC) with level I tumor thrombus through two renal veins, and the renal artery was successfully dissected by retroperitonealscopic approach. Patient 2 had right invasive, immobilized RCC with significant infiltration to IVC and liver. Ligation of renal artery was performed by transperitoneal laparoscopic procedure. Patient 3 had left RCC with level III tumor thrombus and lung metastasis. Ligation of left renal artery and mobilization of peritoneal organs and kidney were performed by transperitoneal laparoscopic surgery. These cases suggest that combined laparoscopic-open surgery for RCC with IVC extension may facilitate early control of arterial blood supply.
\end{abstract}

Keywords: renal cell carcinoma, IVC extension, combined laparoscopic-open surgery

\section{Introduction}

Renal cell carcinoma (RCC) is the most common malignant tumor occurring from renal parenchyma, accounting for $3 \%$ of solid adult neoplasm. ${ }^{1}$ A unique feature of RCC is an intraluminal extension into renal venous circulation, a so-called tumor thrombus. Venous extension from the renal vein to the inferior vena cava (IVC) has been reported in $4 \%-10 \%$ of patients with RCC. ${ }^{2-4}$ Approximately $20 \%-30 \%$ of patients with RCC are reported to have metastasis at the time of diagnosis and distant metastasis after surgical intervention for primary tumor. ${ }^{1,4}$ Although the majority of patients with distant metastasis have unfavorable prognosis, venous extension without metastasis has a more optimistic prognosis. ${ }^{1}$ Indeed, a 5-year cancer-specific survival in such patients is $\sim 60 \%{ }^{5}$ In addition, recently, slight improvement in the prognosis of RCC patients with metastasis and venous extension by treatment with multi-targeted tyrosine kinase inhibitors (TKIs) has been reported. ${ }^{1}$ Therefore, surgical intervention for RCC patients with venous extension should be considered as an option. However, the difficulty of resection increases with the degree of venous extension, which in turn increases the risk of perioperative morbidity and mortality. ${ }^{3}$ Extended tumor thrombus into IVC above the hepatic vein is reported in $<1 \%$ of patients. ${ }^{3}$ The surgical procedure for such cases is technically complex. ${ }^{3-5}$ In addition, clear cell RCC (ccRCC) shows extreme hypervascularity, which increases the potential for significant bleeding during surgery for large RCC with or without tumor thrombus due to the presence of well-developed collaterals. ${ }^{6}$ Therefore, early interception of arterial 
blood supply is a key procedure in nephrectomy; however, early clamping of renal artery may be difficult in some cases as a result of renal arteries located posterior to large hilar RCC, bulky lymphadenopathy, or thickened tumor thrombus.

In this report, we retrospectively reviewed our experience with two recent RCC cases with (internal) IVC extension and a case with direct (external) IVC invasion through adrenal gland. In the initial state of surgical intervention in these three cases, we performed successful early laparoscopic ligation of the renal artery. Our findings are reported in this paper.

\section{Patients and methods}

Two RCC patients with venous extension to IVC and a case of $\mathrm{RCC}$ with direct invasion to IVC underwent radical nephrectomy at our hospital between July 2015 and January 2016. All patients received presurgical axitinib treatment according to the following protocol: All patients received $5 \mathrm{mg}$ axitinib twice daily for 12-16 weeks. Objective response to treatment was assessed by computed tomography (CT) with enhancement at 4 and 12 weeks. Our protocol called for immediate surgical intervention if disease progression was observed at 4 weeks. $^{7}$ The preoperative off-treatment period was 3-4 days. Patient characteristics, clinical stage, and baseline laboratory findings are shown in Table 1 . The performance status of each patient based on Eastern Cooperative Oncology Group criteria was 0 or 1 . Tumor thrombus level was defined according to the classification established by Neves and Zinkcke. ${ }^{8}$ Treatment dose, period, and drug-related adverse events in presurgical axitinib therapy are shown in Table 2. The complications that occurred intraoperatively are shown in Table 3, and postoperative complications were recorded according to the Clavien-Dindo system. ${ }^{9}$

No preoperative transcatheter arterial embolization (TAE) was performed in any of the three cases. During surgery, mobilization of the liver to expose the retrohepatic IVC and management of short hepatic veins as well as exfoliation between tumor and liver were performed by gastroenterological surgeons. Sternotomy and extracorporeal circulation with cardio-pulmonary bypass (CPB) were performed by cardiothoracic surgeons. Pathological result and prognosis are shown in Table 4.

\section{Patient I}

A hypervasucular tumor affecting the right kidney measuring $10 \mathrm{~cm}$ in diameter with tumor thrombus was discovered incidentally in a 53-year-old male. Initial laboratory data were

Table I Clinical characteristics of patients

\begin{tabular}{|c|c|c|c|c|c|c|c|c|c|c|c|c|c|}
\hline \multirow[b]{2}{*}{ Patient } & \multirow[b]{2}{*}{ Age } & \multirow[b]{2}{*}{ Sex } & \multirow[b]{2}{*}{ Laterality } & \multirow[b]{2}{*}{ PS } & \multirow[b]{2}{*}{$\begin{array}{l}\text { Thrombus } \\
\text { level }\end{array}$} & \multicolumn{3}{|c|}{ Clinical stage } & \multicolumn{5}{|c|}{ Laboratory data } \\
\hline & & & & & & $\mathbf{T}$ & $\mathbf{N}$ & $\mathbf{M}$ & $\begin{array}{l}\text { Alb } \\
\text { (g/dL) }\end{array}$ & $\begin{array}{l}\mathrm{Ca} \\
(\mathrm{mg} / \mathrm{dL})\end{array}$ & $\begin{array}{l}\text { LDH } \\
\text { (units/mL) }\end{array}$ & $\begin{array}{l}\text { Hb } \\
(g / d L)\end{array}$ & $\begin{array}{l}\text { CRP } \\
(\mathrm{mg} / \mathrm{dL})\end{array}$ \\
\hline I & 53 & $M$ & $R$ & 0 & 1 & $3 b$ & 0 & 6 & 4.43 & 9.6 & 218 & 16.9 & 0.97 \\
\hline 2 & 63 & $M$ & $\mathrm{R}$ & 0 & - & $\begin{array}{l}4 \\
\text { (adrenal } \\
\text { gland) }\end{array}$ & 0 & 0 & 3.74 & 91 & 149 & 13.6 & 1.69 \\
\hline 3 & 61 & $\mathrm{~F}$ & L & I & III & $3 b$ & 0 & $\begin{array}{l}\text { I } \\
\text { (lung) }\end{array}$ & 2.67 & 10.5 & 368 & 6.9 & 15.5 \\
\hline
\end{tabular}

Abbreviations: PS, Eastern Cooperative Oncology Group performance status; Alb, albumin; Ca, calcium; LDH, lactate dehydrogenase; Hb, hemoglobin; CRP, C-reactive protein.

Table 2 Progress of presurgical treatment and drug-related adverse events

\begin{tabular}{|c|c|c|c|c|c|}
\hline \multirow[t]{2}{*}{ Patient } & \multicolumn{2}{|c|}{ Treatment dose (BID) } & \multirow[t]{2}{*}{ Adverse events } & \multirow{2}{*}{$\begin{array}{l}\text { Treatment period } \\
\text { (days) }\end{array}$} & \multirow{2}{*}{$\begin{array}{l}\text { Off-treatment penoc } \\
\text { (days) }\end{array}$} \\
\hline & $\operatorname{Max}(\mathrm{mg})$ & Min (mg) & & & \\
\hline 1 & 5 & 5 & Hypertension G2 & 81 & 4 \\
\hline \multirow[t]{3}{*}{2} & 5 & 3 & Hypertension G2 & 92 & 3 \\
\hline & & & Hypothyroidism G2 & & \\
\hline & & & Fatigue, Loss of appetite G2 & & \\
\hline 3 & 6 & 5 & Hypertension G2 & 120 & 3 \\
\hline
\end{tabular}

Abbreviations: Max, maximum dose; Min, minimum dose; G, grade.

Table 3 Operative records and perioperative complications

\begin{tabular}{|c|c|c|c|c|c|}
\hline \multirow[t]{2}{*}{ Patient } & \multicolumn{2}{|c|}{ Operation time } & \multirow{2}{*}{$\frac{\text { Bleeding }}{(\mathrm{mL})}$} & \multicolumn{2}{|l|}{ Complications } \\
\hline & Total (min) & Laparoscopy (min) & & Intraoperative & Postoperative (Cevien-Dindo score) \\
\hline I & 494 & 94 & 550 & - & - \\
\hline 2 & 404 & 145 & 4,030 & Blood transfusion & - \\
\hline 3 & 595 & 161 & 1,220 & - & Pleural effusion (Illa) \\
\hline
\end{tabular}


Table 4 Pathological result and prognosis

\begin{tabular}{|c|c|c|c|c|c|c|}
\hline \multirow[b]{2}{*}{ Patient } & \multicolumn{3}{|c|}{ Histopalhological features } & \multicolumn{3}{|l|}{ Prognosis } \\
\hline & Histology & Fuhrman grade & Necrosis (\%) & Follow-up (M) & Outcome (time at event $\mathbf{M}$ ) & Additional treatment \\
\hline I & Clear cell & 2 & 20 & 5 & Alive without disease & - \\
\hline 2 & Clear cell & $2>3$ & 70 & $\mathrm{II}$ & $\begin{array}{l}\text { Alive with contralateral adrenal } \\
\text { metastases (7) }\end{array}$ & Metastasctomy \\
\hline 3 & Clear cell & 2 & 80 & 5 & Alive with bone metastasis (4) & Radiation with TKI \\
\hline
\end{tabular}

Abbreviation: TKI, tyrosine kinase inhibitors.

within normal limits. The tumor thrombus extended to IVC (level I) through two renal veins (Figure 1A-C). However, the ineffectiveness of presurgical treatment prompted the decision to recommend surgery. To prevent perioperative thromboembolism, the IVC filter was placed prior to surgery. The patient was placed in a lateral position, and the laparoscopic procedure employing a retroperitoneal approach was performed for ligating the right renal artery and right lumber veins. We could easily extend the retroperitoneal space, and the target artery was identified and dissected (Figure 1E). The IVC was also exposed, and the right lumber veins were dissected. During this procedure, the majority of the posterior surface of the kidney was easily isolated from the posterior abdominal wall. Next, the patient was repositioned to the supine position, and open surgical procedure was performed through inverted $\mathrm{L}$ incision to the upper abdomen. IVC and bilateral renal veins were exposed, and left lumbar veins and short hepatic veins were dissected. After clamping the caudal IVC, left renal vein, and cephalic IVC, the wall of IVC was cut, and the tumor thrombus with right kidney was removed. The patient recovered without perioperative complications and remained free of recurrence and metastasis for 5 months.

\section{Patient 2}

A 63-year-old male was incidentally diagnosed with right renal tumor during the investigation of left inguinal hernia by CT. A hypervascular tumor measuring $10 \mathrm{~cm}$ in diameter, located at the upper pole of the kidney, was invading directly into the IVC wall through the adrenal gland (Figure $2 \mathrm{~A}-\mathrm{C}$ ). Liver infiltration was also suspected. After 3 months of presurgical axitinib treatment, the tumor decreased in size, and the tumor border to liver appeared clearly. We initiated laparoscopic surgery in the patient in the customary lateral position and employed the transperitoneal approach, because of expected adhesion of tumor and IVC, liver, and posterior abdominal wall, which would increase the difficulty of kidney mobilization. After mobilizing the ascending, transverse colon and duodenum, we approached the renal artery from the caudal side by expanding the space between the lower pole of the kidney and IVC. The
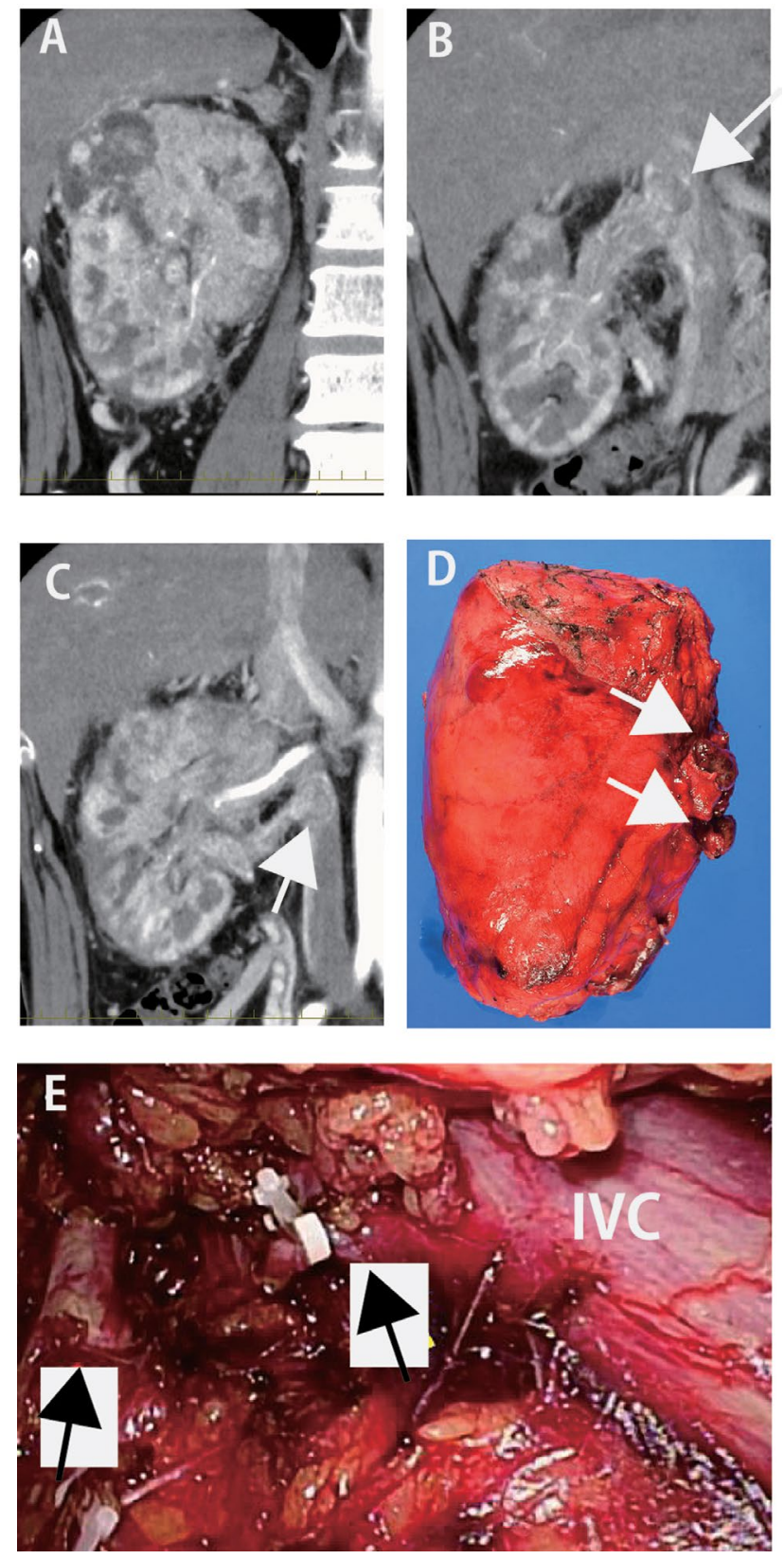

Figure I Computed tomography (CT) findings (A-C), resected specimen (D), and intraoperative laparoscopic findings (E) in Patient I.

Notes: Hypervascular renal tumor (A) with IVC extension through cranial side renal vein (B) and caudal side of renal vein (C). Macroscopic appearance of the specimen is shown (D). Arrowhead indicates the tip of tumor thrombus in each renal veins. Laparoscopic appearance in retroperitoneal approach (E) shows IVC and renal artery (arrowhead, left) and dissected lumber vein (arrowhead, right).

Abbreviation: IVC, inferior vena cava. 

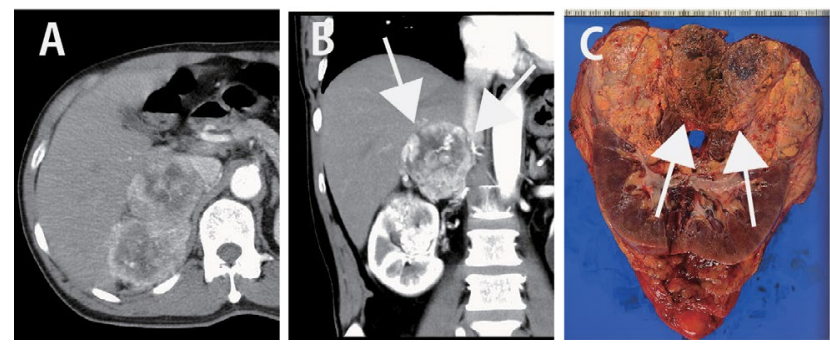

Figure 2 Computed tomography (CT) findings ( $\mathbf{A}$ and $\mathbf{B}$ ) and resected specimen (C) in Patient 2.

Notes: Right renal tumor directly invaded to ipsilateral adrenal gland ( $\mathbf{A}$ and B). Arrowhead indicates cranial border with liver (left) and IVC (right). Surgical specimens are cut longitudinally $(\mathbf{C})$. Yellow-colored tumor is observed at the upper pole of the kidney and adrenal invasion can be seen (arrowheads).

right renal artery was easily detected and ligated. We then ligated the renal veins. The caudal posterior section of the kidney was removed smoothly; however, because the tumor was strongly connected with IVC and liver at the cranial section, we converted to open surgery while maintaining the patient in the same position. Thoracoabdominal incision was made over the bed of the seventh rib and carried to the midline. As partial superficial (capsular) invasion was observed, the liver surface was partly resected. As the tumor invasion to IVC wall was grossly apparent, we performed side clamping to IVC and partially resected the tumor with IVC wall. However, injury of IVC during this procedure required repair of IVC wall by suturing (with blood transfusion). The tumor was partially connected with the diaphragm, thereby requiring repair after the removal of the right kidney. In spite of intraoperative bleeding with blood transfusion, the patient recovered without postoperative complications and has remained free of recurrence and metastasis for 6 months.

\section{Patient 3}

A 61-year-old female presented to our hospital complaining of gross hematuria with palpable left flank mass. Initial laboratory data revealed severe anemia, hypercalcemia, and elevated CRP indicating poor risk according to Memorial Sloan Kettering Cancer Center criteria (Table 1). The patient had hypervasucular tumor at the left kidney measuring $13 \mathrm{~cm}$ in diameter with long venous extension to the suprahepatic IVC (Figure 3A) and left gonadal vein (Figure 3B). In addition, metastases were observed in lungs and hilar lymph nodes (Figure 3C and D). Initially, indication of the nephrectomy was questionable; however, we confirmed that metastases were controllable by presurgical axitinib treatment. Therefore, we considered surgical intervention. After the patient was placed in the lateral position, we began the procedure by laparoscopy to mobilize the spleen, pancreas tail, and descending colon and ligate the left renal artery. Next, we adjusted the patient to the supine position, and open surgical intervention was performed through inverted $\mathrm{T}$ incision to the upper abdomen. IVC and bilateral renal veins were exposed, and the liver was mobilized to expose retrohepatic IVC. As the tumor extension in this case advanced to level III, IVC was secured under the diaphragm, and Pringle maneuver was performed. Under clamping of the right renal vein, hepatoduodenal ligament and IVC (under diaphragm and caudal part to the termination of the right renal vein), a small incision was performed to the anterior surface of IVC; however, we encountered uncontrollable bleeding. Accordingly, we closed the IVC scar, and de-clamped (clamping time was 5 minutes). The segmental resection of infrahepatic IVC is necessary, which, however, would significantly increase the difficulty of reconstruction following removal. For this reason, we performed CPB with deep hypothermic circulatory arrest (DHCA). We performed midline sternotomy in preparation for cannulation to superior vena cava (SVC) and left ventricles to establish $\mathrm{CPB}$ and to commence active cooling toward $20^{\circ} \mathrm{C}$. After initiation of DHCA, IVC was segmentally dissected along with tumor thrombus. Tumor thrombus was covered by latex material and carefully removed by passing it under the superior mesenchymal artery. The left kidney was then extracted completely. The reconstruction of IVC was accomplished with bovine pericardium patch. CPB was reinstituted and re-warming was started. Once normothermia
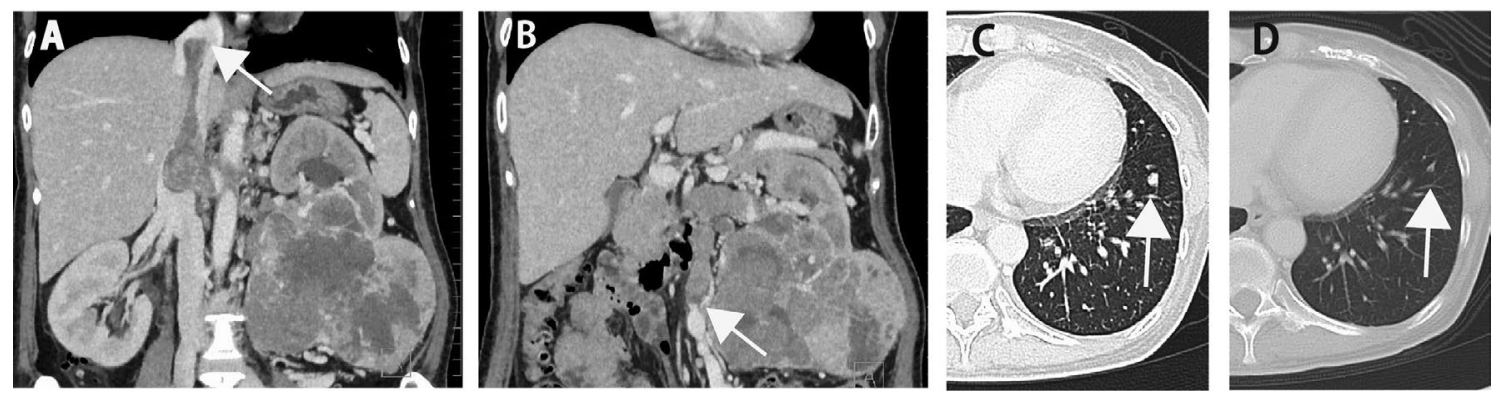

Figure 3 Computed tomography (CT) appearance in Patient 3.

Notes: Left renal tumor extended to IVC $(\mathbf{A})$ and gonadal veins $(\mathbf{B})$. Metastasis of lung $(\mathbf{C})$ was reduced after presurgical treatment $(\mathbf{D})$. Arrowheads indicate the tip of the tumor thrombus. 
was achieved, the patient was weaned from $\mathrm{CPB}$ and decannulated. After ensuring hemostasis, operative scars were closed in ordinary fashion. On the second day post-surgery, the patient complained of dyspnea, which was caused by increased pleural effusion from metastasis. We inserted a drainage tube, which improved the patient's condition. The TKI treatment was initiated 1 month after surgery and has maintained stable disease for 3 months.

\section{Discussion}

Surgical intervention in the treatment of locally advanced RCC may be controversial. Severe mortality $(6.3 \%-10.5 \%)$ is reported in high-level venous extension. In addition, major perioperative complications are reported in $20 \%-30 \%$ of patients with levels $0-$ II venous extension and $34 \%-74 \%$ of patients with levels III-IV. ${ }^{10}$ However, approximately $60 \%$ of RCC patients with venous extension without metastasis respond well to complete removal of tumor with thrombus, ${ }^{5}$ and complete excision with en bloc resection of involved structures, such as IVC wall for large (T4) tumor, may provide a cure. To date, several controversial points regarding the treatment of such cases have been reported. These include the following: 1) control of arterial blood supply in the early phase; 2) liver mobilization technique (collaboration with gastrointestinal surgeon); 3) indication and procedures of CPB with DHCA (collaboration with cardiothoracic surgeon); and 4) indication of cytoreductive nephrectomy for M1 cases. In addition, a well-considered plan created in collaboration with gastroenterological and cardiothoracic surgeons to ensure the inclusion of integrated intraoperative expertise is required to promote successful outcomes.

The advantage of preoperative TAE of renal artery for controlling arterial blood supply has been debated. ${ }^{11-14}$ On the other hand, disadvantages include the risk of pulmonary embolization by necrotic tumor thrombus and postinfarction syndrome with fever and pain. ${ }^{11}$ Ciancio et al reported intraoperative early ligation of right renal artery by "posterior approach," in which the renal artery was ligated after internal mobilization of kidney. ${ }^{12}$ The current approach using laparoscopic surgical procedures achieved the same outcome as TAE and posterior approach in open surgery without risk of TAE-related complication and mobilization of the kidney. In addition, this approach can be performed in a narrow space, in bilateral cases and on various sized tumors. As shown in patient 1 , retroperitoneal approach was very useful for ligation of the right renal artery and lumber veins. Retroperitoneal or transperitoneal laparoscopic nephrectomy combined with open thrombectomy have been reported. ${ }^{13,14}$ In these reports, advantages of laparoscopic approach for procedures in the deep area in the preliminary stage of operation include easier isolation of kidney, ligation of renal artery, isolation of IVC, mobilization of peritoneal organs, positioning of the vessel loop, and dissection of ureter, whereas most delicate procedures such as mobilizing the liver, cutting IVC, removing tumor thrombus, and suturing IVC are suitable for open surgery. ${ }^{13,14}$ In addition, if retroperitoneal approach is possible, this procedure affords easier access to renal artery and IVC than transperitoneal approach because there is no need to mobilize liver, colon, and duodenum in the initial step. ${ }^{13,14}$ In our series, laparoscopic early ligation of renal artery was successfully performed in all cases, by retroperitoneal approach in patient 1 , transperitoneal approach in patient 2 (because mobilization of kidney was impossible), and patient 3 (left RCC with IVC thrombus); and there was no significant perioperative complication with this procedure. Therefore, laparoscopic approach in the early stage of the procedure for cT3b-c-staged RCC may be an option for the control of bleeding. However, evaluation of the potential perioperative outcome advantages of this procedure will require a greater number of cases.

Although the significance of cytoreductive nephrectomy in metastatic RCC (mRCC) is reported, that of surgical intervention in cases with venous extension is still controversial because of the high perioperative complication and mortality rates. ${ }^{15}$ Kwon et al retrospectively analyzed $45 \mathrm{mRCC}$ patients with venous extension. ${ }^{1}$ This study showed that local control may have benefits for survival in the selected cases. In the present case (patient 3 ), our surgical team thoroughly discussed the indication of surgical intervention considering risks and benefits. Because favorable control for primary and metastatic sites was observed by presurgical treatment (Figure 3B and C), we decided that intervention would be appropriate. Although the patient developed dyspnea postoperatively due to increased pleural effusion from metastasis, she recovered after temporary drainage of the thoracic space, and the TKI treatment started smoothly.

We successfully performed nephrectomy combined with laparoscopic and open surgery in three cases of RCC with IVC extension. The advantages of laparoscopic procedure are that it facilitates early ligation of the renal artery and that it allows ease of access to the deep narrow space encountered in various types (size and shape) of renal tumor.

Indication of surgical intervention for $\mathrm{mRCC}$ with IVC thrombus remains controversial; however, cytoreductive nephrectomy may be beneficial in some cases when appropriate targeted therapy is initiated immediately after surgery. The 
current case recovered successfully from the perioperative state; however, careful follow-up is required.

\section{Consent}

Written consent to publish the case details has been provided by the first and second patients in this case series. However, the third patient has since passed away and the authors were unable to contact the next of kin despite exhaustive attempts, therefore the case details have been sufficiently anonymized so that no harm is caused to the patient or their family, the Ethics Committee of Miyazaki University has approved publication of the case details in this instance.

\section{Disclosure}

The authors report no conflicts of interest in this work.

\section{References}

1. Kwon T, Lee JL, You D, et al. Impact of surgery on the prognosis of metastatic renal cell carcinoma with IVC thrombus received TKI therapy. J Surg Oncol. 2014;110(2):145-150.

2. Wang B, Li H, Ma X, Zhang X, et al. Robot-assisted laparoscopic inferior vena cava thrombectomy: different sides require different techniques. Eur Urol. 2016;69(9):1112-1119.

3. Abel EJ, Thompson RH, Margulis V, et al. Perioperative outcomes following surgical resection of renal cell carcinoma with inferior vena cava thrombus extending above the hepatic veins: a contemporary multicenter experience. Eur Urol. 2014;66(3):584-592.

4. Chen YH, Wu XR, Hu ZL, et al. Treatment of renal cell carcinoma with a level III or level IV inferior vena cava thrombus using cardiopulmonary bypass and deep hypothermic circulatory arrest. World J Surg Oncol. 2015;13:159.
5. Blute ML, Leibovich BC, Lohse CM, Cheville JC, Zincke H. The Mayo Clinic experience with surgical management, complications and outcome for patients with renal cell carcinoma and venous tumor thrombus. BJU Int. 2004;94:33-41.

6. González J, Ciancio G. Caval thrombus in conjunction with renal tumors: indication for surgery and technical details. Curr Urol Rep. 2014;15(11):451

7. Mukai S, Kita Y, Fujita N, et al. Presurgical treatment with axitinib in renal cell carcinoma patients with venous extension. Int Canc Conf J. 2014;4 (4): 216-220.

8. Neves RJ, Zinkcke H. Surgical treatment of renal cancer with vena cava extension. Br J Urol. 1987;59:390-395.

9. Dindo D, Demartines N, Clavien PA. Classification of surgical complications: a new proposal with evaluation in a cohort of 6336 patients and results of a survey. Ann Surg. 2004;240(2):205-213.

10. Ebbing J, Wiebach T, Kempkensteffen C, et al. Evaluation of perioperative complications in open and laparoscopic surgery for renal cell cancer with tumor thrombus involvement using the Clavien-Dindo classification. Eur J Surg Oncol. 2015;41(7):941-952.

11. Ciancio G, Vaidya A. Early ligation of the renal artery using the posterior approach: a basic surgical concept reinforced duringresection of large hypervascular renal cell carcinoma with or without inferior vena cava thrombus. MBJU Int. 2003;92(4):488-489.

12. Ciancio G, Vaidya A, Soloway M. Early ligation of the renal artery using the posterior approach: a basic surgical concept reinforced during resection of large hypervascular renal cell carcinoma with or without inferior vena cava thrombus. BJU Int. 2003;92(4):488-489.

13. Hoang AN, Vaporcyian AA, Matin SF. Laparoscopy-assisted radical nephrectomy with inferior vena caval thrombectomy for level II to III tumor thrombus: a single-institution experience and review of the literature. J Endourol. 2010;24(6):1005-1012.

14. Disanto V, Pansadoro V, Portoghese F, Scalese GA, Romano A. Retroperitoneal laparoscopic radical nephrectomy for renal cell carcinoma with infrahepatic vena caval thrombus. Eur Urol. 2005;47(3):352-356.

15. Choueiri TK, Xie W, Kollmannsberger C, et al. The impact of cytoreductive nephrectomy on survival of patients with metastatic renal cell carcinoma receiving vascular endothelial growth factor targeted therapy. J Urol. 2011;185(1):60-66.
Research and Reports in Urology

\section{Publish your work in this journal}

Research and Reports in Urology is an international, peer-reviewed, open access journal publishing original research, reports, editorials, reviews and commentaries on all aspects of adult and pediatric urology in the clinic and laboratory including the following topics: Pathology, pathophysiology of urological disease; Investigation and treatment of

\section{Dovepress}

urological disease; Pharmacology of drugs used for the treatment of urological disease. The manuscript management system is completely online and includes a very quick and fair peer-review system, which is all easy to use. Visit http://www.dovepress.com/testimonials.php to read real quotes from published authors. 